\title{
Psycho-Education Intervantion Program Among Women with Breast Cancer
}

ISSN: 2637-773X

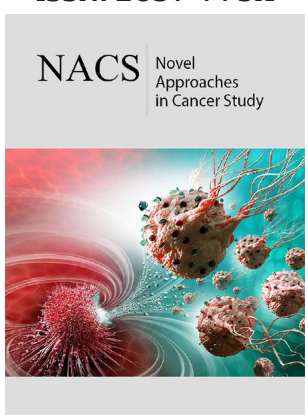

*Corresponding author: Murad Abdul Raheem Younis, Department of Assistance Medical Science, Coordinator of Quality and Academic Accreditation, Tabuk 47512, Saudi Arabia

Submission: 祭 June 14, 2021

Published: 倫June 24, 2021

Volume 6 - Issue 1

How to cite this article: Murad Abdu Raheem Younis. Psycho-Education Intervantion Program Among Women with Breast Cancer. Nov Appro in Can Study. 6(1). NACS.000627. 2021. DOI: 10.31031/NACS.2021.06.000627

Copyright@ Murad Abdul Raheem Younis, This article is distributed under the terms of the Creative Commons Attribution 4.0 International License, which permits unrestricted use and redistribution provided that the original author and source are credited.

\author{
Murad Abdul Raheem Younis* \\ Department of Assistance Medical Science, Saudi Arabia
}

\section{Introduction}

Women with breast cancer face challenges in different aspects of their lives, as the drastic changes in their psychological needs can be distressful. With the intention of helping these women to cope after the diagnosis, there has been an emphasis in providing psychological support and interventions to enhance Quality of Life (QOL) and survival rate. It is imperative to investigate the psychological and psychosocial factors interrupting the quality of life and wellbeing in female cancer patients with the intention of providing them with proper treatment and therapeutic intervention. Psycho-education is a form of therapeutic intervention for patients and their families. One of the most operative psycho-social approaches to cancer patients is psycho-education Dastan [1], Younis et al. [2]. Psycho-education is important as it is a process that provides education and information to individuals who seek or receive mental health services, such as individuals that are seriously or terminally ill and also their family members. The program normally includes health education, stress management, coping skills, and psychological support.

A psycho-education intervention program's objective is to help people to understand a patient's mental situation and adapt to the changes caused by the illness. Understanding the types of challenges can help patients, in this case, women with breast cancer, to have better knowledge of the physical and emotional changes they will face, identify their own strengths to help them cope better with the treatment and have more control over their own health.

Psychosocial support is found effective to improve breast cancer indications and psychoeducational support in order to improve emotional health within six month following post intervention Nishimoto [3]. Takahasi et al. stated that psychopathological symptoms, for example anxiety and depression, are common among patients with cancer. Their findings substantiated a study by Avelar et al. who revealed that anxiety and depression levels among pre- and post-surgery in women with breast cancer were notably maintained after pyschoeducational intervention.

In general, psycho-education intervention should aid women with breast cancer in coping with their stressors by enhancing the score of QOL domains and encouraging adaptive coping outcomes. A Coping strategy can help patients diagnosed with breast cancer to decrease psychological distress like anxiety and depression, and to enhance their QOL. However, while the association between QOL, coping strategies and psychological distress has been studied extensively, little research has been undertaken to examine the effectiveness of a psychoeducation intervention program in enhancing reappraisal of stressful situations among Jordanian women diagnosed with breast cancer and undergoing different treatments. Limited data are available regarding coping strategies, and there is no program as an intervention to perceive QOL, coping strategy and psychological distress among Jordanian women with breast cancer Younis et al. [4]. 
Besides, psycho-education intervention is significant in improving well-being and reducing psychological distress like depression, stress, and anxiety among Jordanian breast cancer survivors Younis et al. [2,5]. Psycho-education therapy assists women with breast cancer pertaining to these effects of the disease on their QOL, psychological distress, and how to select a proper coping strategy. Psycho-education has improved coping with the diagnosis, effective management of daily stressors by using problem-focused coping strategy (adaptive coping) and increase the overall QOL of Jordanian women breast cancer survivors. Younis et al. [2]. Most importantly, family members need to be included in the programs to help counter the undesirable effects of the recurring disease on their mental fitness, and to allow them to continuously act as operative caregivers Younis et al. [2] \& Laurel et al. [6].

\section{References}

1. Dastan NB, Buzlu S (2012) Psychoeducation intervention to improve adjustment to cancer among Turkish stage I-II breast cancer patients: A randomized controlled trial. Asian Pacific Journal of Cancer Prevention 13(10): 5313-5318.
2. Younis M, Norsa'adah B, Othman A (2021) Effectiveness of psychoeducation intervention programme on coping strategies among jordanian women diagnosed with breast cancer: A randomised controlled trial. Breast Cancer (Dove Med Press) 13: 285-297.

3. Nishimoto A (2014) Effectiveness of psychoeducational support on quality of life in early-stage breast cancer patients: A systematic review and meta-analysis of randomized controlled trials. Quality of Life Research 23(1): 21-30.

4. Younis MAR, Norsa'adah B, Othman A, Shaqarin Y, Alqudah H, et al. (2020) Psychological distress impact and associated factors among Jordanian women diagnosed with breast cancer. Medical Science 24(104): 2610-2619.

5. Younis MAR, Othman A, Norsa'adah B (2020) Psychoeducational intervention program for women with breast cancer: A systematic review and meta-analysis. Medical Science 24(104): 2110-2118.

6. Laurel LN, Mood D, Kershaw T, Schafenacker A, Mellon S, et al. (2002) Quality of life of women with recurrent breast cancer and their family members. Journal of Clinical Oncology 20(19): 4050-4064. 